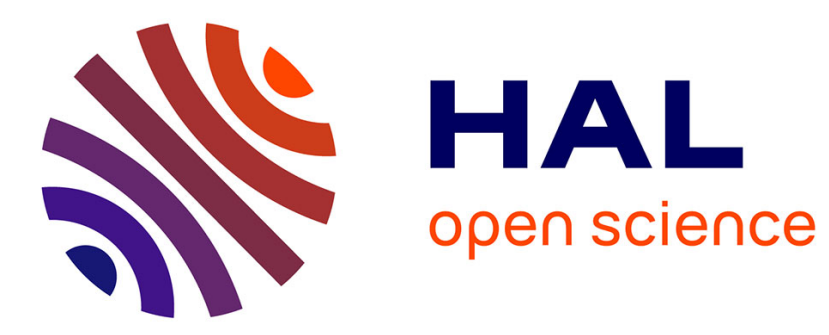

\title{
Theoretical insights into the formation and stability of radical oxygen species in cryptochrome
}

\author{
Padmabati Mondal, Miquel Huix-Rotllant
}

\section{To cite this version:}

Padmabati Mondal, Miquel Huix-Rotllant. Theoretical insights into the formation and stability of radical oxygen species in cryptochrome. Physical Chemistry Chemical Physics, 2019, 21, pp.88748882. 10.1039/C9CP00782B . hal-02088383

\section{HAL Id: hal-02088383 \\ https://hal-amu.archives-ouvertes.fr/hal-02088383}

Submitted on 2 Apr 2019

HAL is a multi-disciplinary open access archive for the deposit and dissemination of scientific research documents, whether they are published or not. The documents may come from teaching and research institutions in France or abroad, or from public or private research centers.
L'archive ouverte pluridisciplinaire HAL, est destinée au dépôt et à la diffusion de documents scientifiques de niveau recherche, publiés ou non, émanant des établissements d'enseignement et de recherche français ou étrangers, des laboratoires publics ou privés. 


\title{
Journal Name
}

\section{ARTICLE TYPE}

Cite this: DOI: $10.1039 / x x x x x x x x x x$

\section{Theoretical insights into the formation and stability of radical oxygen species in cryptochrome}

\author{
Padmabati Mondal, ${ }^{* a}$ and Miquel Huix-Rotllant ${ }^{* a}$
}

Received Date

Accepted Date

DOI: $10.1039 / x x x x x x x x x x$

www.rsc.org/journalname

\begin{abstract}
Cryptochrome is a blue-light absorbing flavoprotein containing a flavin adenine dinucleotide (FAD) cofactor. FAD can accept up to two electrons and two protons, which can be subsequently transferred to substrates present in the binding pocket. It is well known that reactive oxygen species are generated when triplet molecular oxygen is present in the cavity. Here, we investigate the formation and stability of radical oxygen species in Drosophila melanogaster cryptochrome using molecular dynamics simulations and electronic structure calculations. We find that superoxide and hydroxyl radicals in doublet spin states are stabilized in the pocket due to attractive electrostatic interactions and hydrogen bonding with partially reduced FAD. These finding validate from a molecular dynamics perspective that [FAD ${ }^{\bullet-}$ $\mathrm{HO}_{2}^{*}$ ] or $\left[\mathrm{FADH}{ }^{\bullet}-\mathrm{O}_{2}^{--}\right.$] can be alternative radical pairs at the origin of magnetoreception.
\end{abstract}

\section{Introduction}

Cryptochromes are a type of flavoproteins that absorb blue-UV/A light. ${ }^{1}$ They are found throughout the biological kingdom (microbes, plants, animals, etc.) ${ }^{2,3}$, involved in several biological processes like plant growth or entrainment of the circadian clock in animals. ${ }^{1,4-7}$ Cryptochromes contain vitamin $\mathrm{B}_{2}$ acting as photoactive oxidizing agent, found either in the form of flavin mononucleotide (FMN) or flavin adenine dinucleotide (FAD). The vitamin $B_{2}$ cofactor, also known as riboflavin, is common to all flavoproteins, and it is responsible for their functionality. It consists of a lumiflavin moiety acting as chromophore and a pentane2,3,4-triol side chain. Lumiflavin is a good oxidizing agent and a strong base, accepting up to two electrons and two protons. The oxidizing potential is increased upon light absorption.

Initially, the cofactor is usually found in its fully oxidized form. The oxidized FAD $\left(\mathrm{FAD}_{o x}\right)$ can then be reduced to the anionic radical semi-quinone form $\left(\mathrm{FAD}^{\bullet-}\right)$ via electron transfer from a residue near the flavin chromophore. In cryptochromes, the most widely accepted mechanism is that this electron is transferred from a nearby tryptophan that is concomitantly oxidized to $\operatorname{TrpH}^{\bullet+} .{ }^{8}$ The anionic radical $\mathrm{FAD}^{\bullet-}$ is a strong base that can accept a proton to generate the neutral semi-quinone $\mathrm{FADH}^{\bullet}$. The origin of this proton depends on the type of cryptochrome. For arabidopsis thaliana cryptochrome, an aspartic acid donates a proton to FAD and $\operatorname{TrpH}^{\bullet+}$ subsequently transfer another proton

\footnotetext{
${ }^{a}$ Aix-Marseille Univ, CNRS, ICR, Marseille, France.

E-mail: padmabati.mondal@gmail.com; miquel.huixrotllant@univ-amu.fr

$\dagger$ Electronic Supplementary Information (ESI) available: [details of any supplemen-
} tary information available should be included here]. See DOI: 10.1039/b000000x/ to the aspartate, ending up in a neutral tryptophanyl radical Trp• and a neutral semi-quinone FADH ${ }^{\bullet}{ }^{8}$ For Drosophila melanogaster cryptochrome, the participating residues in this proton transfer process have not yet been clearly identified. The $\mathrm{FADH}^{\bullet}$ can still act as a photooxidizer and a photobase, absorbing in the visible region, leading to the anionic reduced form $\mathrm{FADH}^{-}$when accepting one electron and the neutral reduced form $\mathrm{FADH}_{2}$ when sequentially accepting a proton. ${ }^{9-11}$

Cryptochromes have been proposed as plausible candidates for explaining the light-dependent magnetic field sensors, a capacity of certain animals to guide by perceiving the geomagnetic field (so-called magnetoreception). ${ }^{9-11}$ Spin-correlated radical pairs between flavin and tryptophan (FADH ${ }^{\bullet}-\mathrm{Trp}^{\bullet}$ ) generated by photoreduction in plant cryptochrome have been reported to be at the origin of magnetoreception. ${ }^{8-10,12}$ This is known as the radical pair mechanism of magnetoreception (hereafter referred as "conventional" radical pair). A recent study showed that tryptophan triad might not be necessary for magnetoreception in Drosophila melanogaster, ${ }^{13}$ opening up the route for alternative radical pairs in certain cryptochromes. It has been proposed FADH ${ }^{\bullet}$ can form a radical pair with oxygen superoxide $\left(\mathrm{O}_{2}^{--}\right)$in lieu of tryptophan. ${ }^{14,15}$ Recent theoretical studies indicate that the $\mathrm{FADH}^{\bullet}-\mathrm{O}_{2}^{\bullet-}$ radical pair could resist better to decoherence phenomena, thus being a better candidate for magnetoreception than the tryptophan-flavin pair. ${ }^{16,17}$

Superoxide can be formed in cryptochromes during the reoxidation reaction of the fully reduced anionic form of FAD in the dark environment $\left(\mathrm{FADH}^{-} \rightarrow \mathrm{FADH}^{\bullet} \rightarrow \mathrm{FAD}_{o x}\right)$. There are experimental evidences that reactive oxygen species (ROS) can 


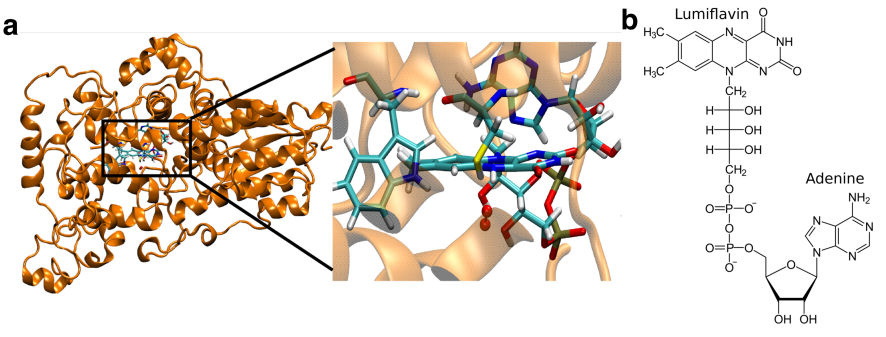

Fig. 1 (a) Structure of dmCry- $\mathrm{O}_{2}$ system. The zoomed active site of dmCry with $F A D_{o x}-O_{2}$ is shown as an inset in the right hand side (b) Structure of flavin adenine dinucleotide.

be present in the active site of cryptochrome. ${ }^{18-21}$ Combined molecular dynamics simulation and kinetic studies confirm that indeed oxygen molecule can access the flavoprotein binding site via multiple pathways facilitating catalytic oxidation procedure. ${ }^{22}$ While several studies focused on the photo-reduction reaction, $8,11,12,23-25$ not much attention has been given to the study of the re-oxidation reaction. ${ }^{18,26}$

Here, we perform a thorough molecular dynamics study of different oxygen species including the ROS within the Drosophila melanogaster cryptochrome depending on the redox and protonation states of flavin chromophore. In order to explain the oxidation state of oxygen complexed with different redox forms of FAD, multi-configurations electronic structure calculations were performed. We find that the ROS stay close to the flavin chromophore via strong attractive electrostatic interactions or hydrogen bonding, while the triplet molecular oxygen diffuses out of the flavin pocket when FAD is reduced. These results support the existence of $\left[\mathrm{FAD}^{\bullet-}-\mathrm{HO}_{2}^{\bullet}\right]$ or $\left[\mathrm{FADH}^{\bullet}-\mathrm{O}_{2}^{\bullet-}\right]$ as alternative radical pair involving superoxide as the basis for the magnetoreception in Drosophila melanogaster cryptochrome.

\section{Computational Details}

The initial protein structure (with FAD co-factor, see Figure 1) is taken from the chain B of the crystal structure of Drosophila melangoster cryptochrome (dmCry), PDBID:4K03. In order to obtain the initial structure of dmCry $-\mathrm{O}_{2}$ complex, an oxygen molecule has been placed in the active site according to the gas-phase optimized structure of $\mathrm{FAD}-\mathrm{O}_{2}$ complex obtained at the wB97x-D/6-31G* level. ${ }^{27,28}$ Molecular dynamics simulations based on several random initial conditions of the FAD- $\mathrm{O}_{2}$ complex have been performed to determine the stability of the results (see Supporting Information). The initial dmCry- $\mathrm{O}_{2}$ structure with $\mathrm{FAD}_{o x}-\mathrm{O}_{2}$ (A) is neutralized with $13 \mathrm{Na}^{+}$ions and solvated in a $105 \times 105 \times 105 \AA^{3}$ water box rendering the total system size of 114,012 atoms. The other protonation states of the dmCry- $\mathrm{O}_{2}$ complex have been generated from the initial dmCry $-\mathrm{O}_{2}$ structure of the fully oxidized form. All molecular dynamics (MD) simulations were performed using Gromacs software. ${ }^{29}$ The solvated and ionated dmCry- $\mathrm{O}_{2}$ structures were then minimized and equilibrated using NVT (for 200 ps) and NPT (1 ns) ensembles at $\mathrm{T}=300 \mathrm{~K}$. A production run of 10 ns was performed for each system. The electrostatic interactions between FAD and oxygen are represented by the set of charges calculated considering oxygen as superoxide, hydroperoxyl radical or triplet as well as corresponding redox and spin state of FAD.

Charmm27 force fields ${ }^{30,31}$ have been employed for the protein scaffold. The bonded, angular, dihedral and van der Waals parameters of FAD species are taken from CGENFF database and are considered to be the same for all possible redox and protonated states. ${ }^{32}$ The bonded parameters for oxygen species are taken from density functional theory employing the rangeseparated exchange-correlation functional with dispersion correction (wB97x-D) and 6-31G* atomic basis set. These quantum chemical calculations have been performed using GAUSSIAN 16. ${ }^{33}$ The electrostatic part of the force fields for the $\mathrm{FAD}$ and $\mathrm{O}_{2}$ species has been reparameterized based on Mulliken atomic charges obtained from density functional theory employing wB97x-D functional and 6-31G* atomic basis set. ${ }^{27,28}$ Since Charmm force field relies on Mulliken atomic charges as the initial guess for the protein electrostatic interactions, ${ }^{34,35}$ we have employed them for the FAD and oxygen species to be consistent. To assess the validity of the force field, we have compared molecular dynamics simulations using our Mulliken atomic charges and Merz-Kollman electrostatic potential fitting atomic charges, leading to similar evolution for the $\mathrm{C}_{4 a}$-OO1 distance, indicating a similar description of the electrostatic interactions between oxygen and FAD (see Supporting Information). Mulliken charges for the optimized structure for the different redox states of FAD, triplet oxygen ${ }^{3} \mathrm{O}_{2}$ and doublet superoxide ${ }^{2} \mathrm{O}_{2}^{\bullet-}$ and hydroxyl ${ }^{2} \mathrm{HO}_{2}^{\bullet}$, according to their total charge and spin multiplicity have been used as the electrostatic force fields parameters for the MD simulations. Hereafter, the superscripts on the left indicate the spin multiplicity of the fragment.

The electronic structure calculations for the radical and anionic $\mathrm{FAD}-\mathrm{O}_{2}$ systems were performed using state-averaged Complete Active Space Self Consistent Fields (SA-CASSCF) method with second-order perturbation theory correction (CASPT2). The state-averaged for five electronic states using an active space of $(11,12)$ for radicals and $(12,12)$ for the rest. For multiconfiguration calculations, the ANO-RCC-VDZP basis set has been employed. ${ }^{36}$ For these calculations, OpenMolcas package has been used. ${ }^{37}$

\section{Results and discussion}

In Figure 2a, we summarize the redox forms of FAD starting from the blue-light absorption of $\mathrm{FAD}_{o x}$, supposing that the electron is donated by a residue near the cavity. ${ }^{9,10}$ When triplet oxygen is present inside the cavity, the $\mathrm{FAD}-\mathrm{O}_{2}$ complex can exist in several spin states (see Figure $2 \mathrm{~b}$ ). Initially, the complex is in a total triplet state ${ }^{3}\left[\mathrm{FAD}_{\mathrm{ox}}-\mathrm{O}_{2}\right]$ composed of singlet ${ }^{1} \mathrm{FAD}_{\mathrm{ox}}$ (fully oxidized FAD) and triplet molecular oxygen ${ }^{3} \mathrm{O}_{2}$. Upon reduction of FAD by tryptophan, the complex can exist either in total doublet ${ }^{2}\left[\mathrm{FAD}^{\bullet-}-\mathrm{O}_{2}\right]$ or quartet state ${ }^{4}\left[\mathrm{FAD}^{\bullet-}-\mathrm{O}_{2}\right]$, depending on the relative orientation of oxygen spin with respect to the FAD electron. Subsequently, a proton can be transferred to neutralize this charge generating again a doublet ${ }^{2}\left[\mathrm{FADH}^{\bullet}-\mathrm{O}_{2}\right]$ or a quartet ${ }^{4}\left[\mathrm{FADH}^{\bullet}-\mathrm{O}_{2}\right]$ with the partially reduced neutral semi-quinone ${ }^{2} \mathrm{FADH}^{\bullet}$. When a second electron is transferred, the complex can 
a

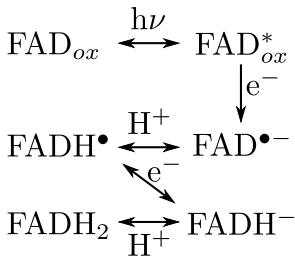

b $\quad{ }^{3}\left[\mathrm{FAD}_{o x}-\mathrm{O}_{2}\right] \mathbf{A}$

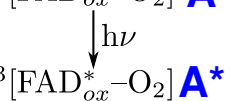

Fig. 2 (a) Redox and acid-base reactions path of flavin adenine dinucleotide. The reduction can be induced photochemically by absorption of blue light by FAD. (b) Redox and electron exchange reactions path involving FAD and oxygen. For convenience, each system is labelled with a one letter tag (A-K) in blue.

be in singlet ${ }^{1}\left[\mathrm{FADH}^{-}-\mathrm{O}_{2}\right]$ or triplet ${ }^{3}\left[\mathrm{FADH}^{-}-\mathrm{O}_{2}\right]$. Here, we have only considered FAD as the electron/proton acceptor and oxygen as a spectator. However, oxygen can re-oxidize reduced FAD $\left(\mathrm{FADH}^{\bullet}\right.$ or $\mathrm{FADH}^{-}$) and accept protons. This would imply an intramolecular reaction that leaves the total charge and spin of the complex unchanged. The resulting structures produce the ROS, ${ }^{2} \mathrm{O}_{2}^{\bullet-}$ or hydroxyl ${ }^{2} \mathrm{HO}_{2}^{\bullet}$ radicals.

The main reaction paths involving FAD and $\mathrm{O}_{2}$ are summarized in Figure $2 \mathrm{~b}$. The initial state of cryptochrome is ${ }^{3}\left[\mathrm{FAD}_{o x}-\mathrm{O}_{2}\right]$ (A). When photoexcited $\left(\mathbf{A}^{*}\right)$, it can be reduced by a nearby tryptophan. When the semireduced FAD- $\mathrm{O}_{2}$ complex is in quartet (B), molecular oxygen is a spectator, and the next proton $(\mathbf{B} \rightarrow \mathbf{C})$ and the subsequent electron transfer $(\mathbf{C} \rightarrow \mathbf{F})$ can only be accepted by FAD. Instead, when reduced anionic FAD is forming a doublet with $\mathrm{O}_{2}$ (D), the next proton and electron can be accepted by $\mathrm{FAD}^{\bullet-}(\mathbf{D} \rightarrow \mathbf{E} \rightarrow \mathbf{F})$. There is though the possibility that superoxide is formed (G) and thus an equivalent superoxide channel exists $(\mathbf{G} \rightarrow \mathbf{H} \rightarrow \mathbf{I})$. Finally, when a proton is transferred to oxygen, two hydroxyl radicals can be formed with oxidized FAD (J) and partially reduced FAD (K). The systems $\mathbf{D}$ and $\mathbf{E}$ (total doublet with triplet oxygen and radical FAD) produce the same partial charges as $\mathbf{B}$ and C, respectively. They are only distinguished by the orientation of the triplet spin, either parallel to the radical spin on FAD (quartet) or anti-parallel (doublet). Therefore we performed the simulations for the simpler quartet system $\mathbf{B}$ and $\mathbf{C}$, assuming them equivalent to the system $\mathbf{D}$ and $\mathbf{E}$, respectively, in the molecular dynamics simulation.

\subsection{Presence of oxygen species in the binding pocket}

A fundamental issue to investigate the role of $\mathrm{O}_{2}$ in cryptochromes is to know which of these species are stabilized in the active site, near the flavin moiety. For this purpose, we have investigated several flavin-oxygen complexes for different spin and protonation states. For this study, we have defined the active site as the cavity around FAD by a sphere with radius of $5 \AA{ }^{22}$ Along the trajectories, we collect the distances between the 001 atom of the oxygen and the C4a atom (the FAD atom known to interact with oxygen species) ${ }^{26}$ during the $10 \mathrm{~ns}$ MD simulations.

The time-evolution of distances is shown in Figure 3. The C4a-OO1 distance for the systems with triplet oxygen (Figure 3a)
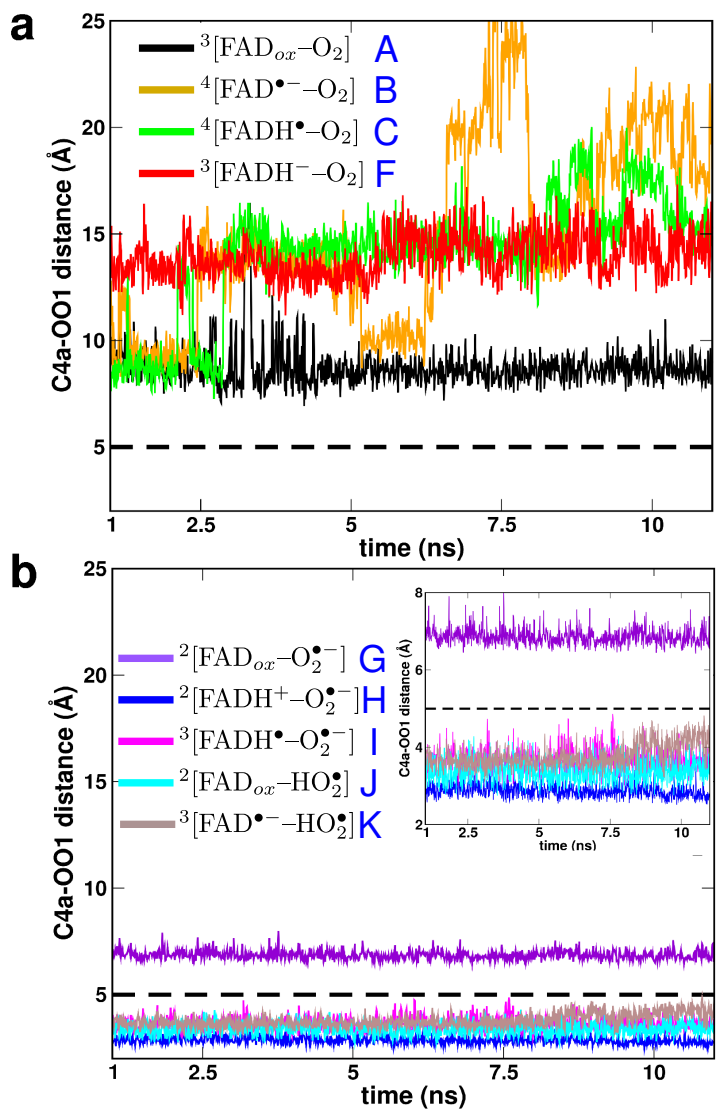

Fig. 3 The distance between $\mathrm{C} 4 \mathrm{a}$ atom of the chromophore and $\mathrm{OO} 1$ atom of the oxygen species for $10 \mathrm{~ns}$ of MD simulation of each system. The horizontal dashed black line refers to the radius of the C4a cavity. The distances for the dynamics of neutral and radical oxygen species are separated in the upper (a) and lower panel (b), respectively. The inset of panel b shows the $\mathrm{C} 4 \mathrm{a}-\mathrm{OO} 1$ distances for radical oxygen species in a optimized $y$-scale. 


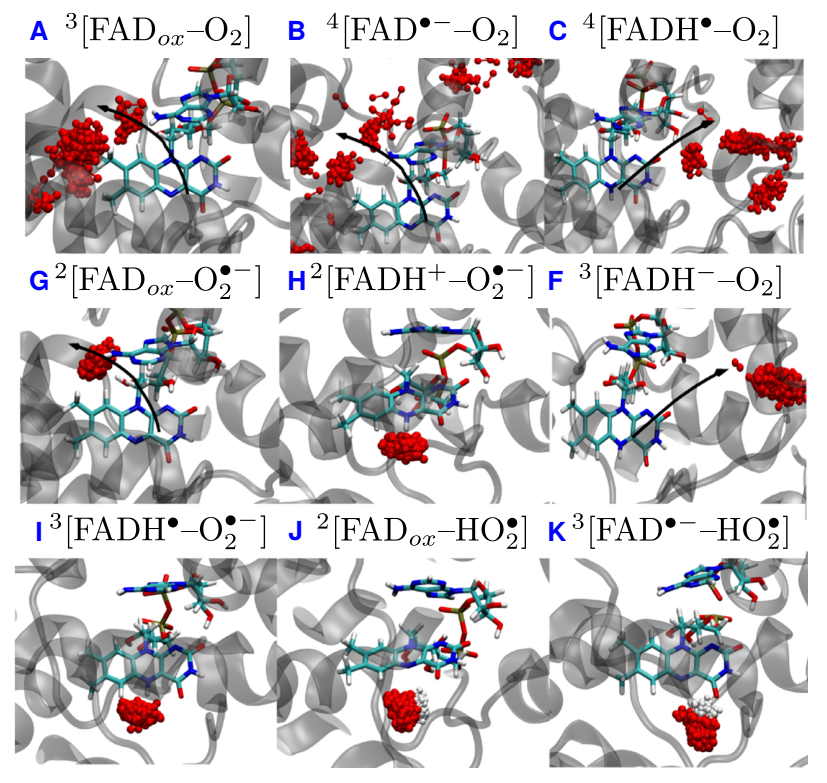

Fig. 4 Specific position of the oxygen species (in red CPK representation) in dmCry- $\mathrm{O}_{2}$ for all systems (A to $\mathrm{K}$ ) as clusters from 200 snapshots taken at $50 \mathrm{ps}$ intervals during $10 \mathrm{~ns}$ of simulation. The black arrows indicate the diffusing pathway of oxygen species.

show in general a rather repulsive interaction with FAD. The distance between oxygen and FAD depends strongly on the redox state of FAD. In the initial $\mathrm{FAD}_{o x}$ form, molecular oxygen is stabilized around $3 \AA$ away from the binding pocket. This is a steady value with small fluctuations on the average distance, in accordance with the fact that oxygen is present inside the protein. ${ }^{22,26}$ For the radical forms $\left(\mathrm{FAD}^{\bullet-}\right.$ and $\left.\mathrm{FADH}{ }^{\bullet}\right)$, oxygen diffuses away from the binding pocket. Large fluctuations are observed for these C4a-OO1 distances, indicating a strong repulsive interaction between reduced FAD and ${ }^{3} \mathrm{O}_{2}$, the distance of which can be as large as $20 \AA$. Finally, for the anionic reduced form $\left(\mathrm{FADH}^{-}\right)$, oxygen is stabilized on an average at around $9 \AA$ far from the pocket boundary. This suggest a slow reoxidation reaction for $\mathrm{FADH}^{-} \rightarrow \mathrm{FADH}^{\bullet}$ and an even slower $\mathrm{FADH}^{\bullet} \rightarrow \mathrm{FAD}_{o x}$, in accordance with kinetic experiment. ${ }^{18}$ Upon electron transfer from reduced flavin to oxygen, flavin is reoxidized and superoxide is formed. Subsequently, FAD can be again reduced absorbing a second photon thus forming several radical pairs with either superoxide or hydroxyl radicals. In Figure 3b, we observe that these radical oxygen species are all stabilized inside the binding pocket, very close to FAD. On average, these distances are around 3-4 $\AA$ and undergo very low fluctuation. This indicates a strong attractive electrostatic interaction of oxygen superoxide and hydroxyl radicals with the residues in the binding pocket. This would suggest that absorbing one photon is required to generate superoxide and another photon can generate a radical pair and stabilizes superoxide inside the pocket.

In order to clarify the interaction between the oxygen species and the cavity, we analyze the specific binding location or specific diffusion pathway of the oxygen species. For this purpose, we performed a cluster plot of oxygen species from 200 snapshots

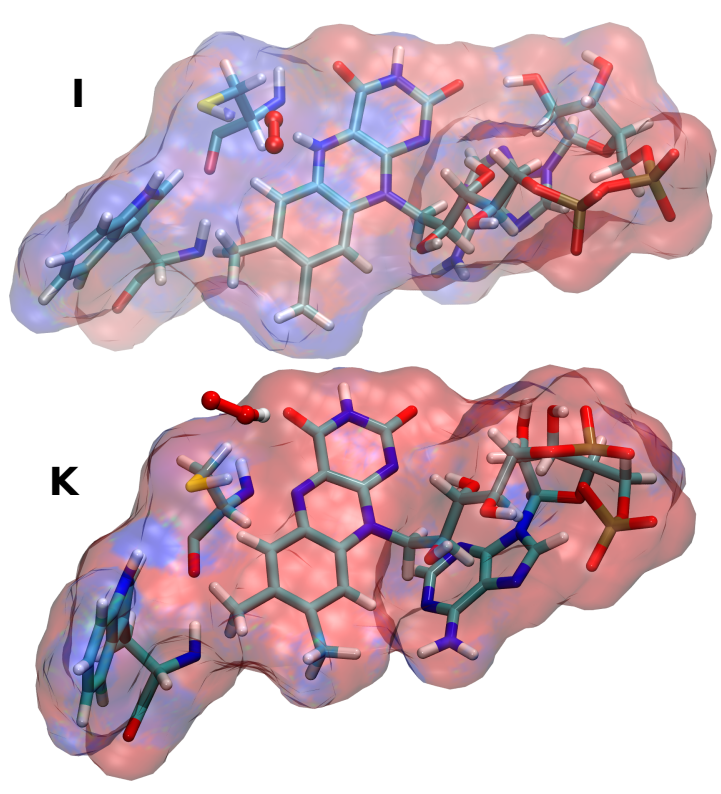

Fig. 5 The electrostatic potential of FADH ${ }^{\bullet}$, Trp420 and Cys 416 felt by the superoxide and hydroperoxyl radical for ${ }^{3}\left[\mathrm{FADH}^{\bullet-} \mathrm{O}_{2}^{--}\right](\mathrm{I})$ and ${ }^{3}\left[\mathrm{FAD}^{\bullet-}-\mathrm{HO}_{2}^{*}\right](\mathrm{K})$ system. The electrostatic potential (ESP) is calculated using Adaptive Poisson-Boltzmann Solver (APBS) ${ }^{38}$ in gas phase. The ESP is shown in a RGB (Red-Green-Blue) color scale in a range of -0.05 to $0.05 k_{b} T / e$.

taken at $50 \mathrm{ps}$ intervals during $10 \mathrm{~ns}$ trajectories of dmCry- $\mathrm{O}_{2}$ complex. The results are shown in Figure 4. One can observe that the position of the oxygen species is different depending on whether it is a ${ }^{3} \mathrm{O}_{2}$ or ${ }^{2} \mathrm{O}_{2}^{--}$or a $\mathrm{HO}_{2}^{\bullet}$. When oxygen is in superoxide form, it stays near the flavin stabilized by the attractive electrostatic interaction dominated by the hydrogen in the N5 position i.e. only in case of ${ }^{2}\left[\mathrm{FADH}^{+}-\mathrm{O}_{2}^{\bullet-}\right](\mathrm{H})$ and ${ }^{3}\left[\mathrm{FADH}^{\bullet}-\right.$ $\mathrm{O}_{2}^{\bullet-}$ (I) (see Figure 5). The $\mathrm{HO}_{2}^{\bullet}$ species resides always close to the FAD due to electrostatic interaction between the $\mathrm{H}$ of $\mathrm{HO}_{2}^{*}$ and N5 of FAD (in case of ${ }^{2}\left[\mathrm{FAD}_{o x}-\mathrm{HO}_{2}^{*}\right](\mathbf{J})$ and ${ }^{3}\left[\mathrm{FAD}^{\bullet-}-\mathrm{HO}_{2}^{*}\right]$ (K). On the contrary, triplet molecular oxygen diffuses out of the flavin pocket and that is independent of the redox and protonated states of the FAD. We observe only two distinct paths (shown with black arrows in Figure 4) via which the oxygen species moves outside of the lumiflavin pocket. After diffusing out from the flavin pocket, triplet oxygen is either stabilized or it hops between very few cryptochrome pockets except in the case of ${ }^{4}\left[\mathrm{FAD}^{\bullet-}-\mathrm{O}_{2}\right]$ (B) where the triplet oxygen is hopping between several pockets.

Radical superoxide and hydroxyl radicals are essentially stabilized by electrostatic interactions and hydrogen bonding. To show this, we have computed the electrostatic potential due to the FAD species, Trp420 and Cys416 felt by the oxygen species for the final stable structure of ${ }^{3}\left[\mathrm{FADH}^{\bullet}-\mathrm{O}_{2}^{\bullet-}\right]$ (I) and ${ }^{3}\left[\mathrm{FAD}^{\bullet-}-\mathrm{HO}_{2}^{\bullet}\right]$ (K) which are shown in Figure 5. The electrostatic potential (ESP) is calculated using Adaptive Poisson-Boltzmann Solver (APBS) ${ }^{38}$ implemented in vmd ${ }^{39}$. The ESP is shown in a RGB (Red-GreenBlue) color scale in a range of -0.05 to $0.05 k_{b} T / e$. For system I, we can see that a positive potential (blue), with contribution from FAD, Trp420 and Cys416 group and dominated by the contribution from $\mathrm{N} 5 \mathrm{H}$ group of lumiflavin, is created in the region around 
the superoxide (shown in CPK representation) and therefore the superoxide, having negative electrostatic potential due to its' negative charge, is highly stabilized in that region due to strong electrostatic interaction. On the other hand, in system $\mathbf{K}$, the negative ESP (red) around the $\mathrm{FAD}^{\bullet-}$ stabilizes the $\mathrm{HO}_{2}^{\bullet}$ radical (shown in $\mathrm{CPK}$ representation in the lower panel of Figure 5) since $\mathrm{HO}_{2}^{\bullet}$ itself generate a positive electrostatic potential around the $\mathrm{H}$ atom. Finally, the triplet oxygen, having zero partial charge, does not feel any attractive potential and therefore can freely move in and around the binding pocket.

The hydrogen bonding between FAD and radical oxygen species stabilizes further ROS in the binding pocket. We observe that in fact hydrogen bonding induces a stronger binding of oxygen than the pure non-bonded electrostatic interactions. Indeed, the stabilization of hydroxyl radical strongly depends on the initial conditions, whereas this is not observed for the superoxide or triplet oxygen species. The $\mathrm{HO}_{2}^{\bullet}$ stays within the cavity only when the simulation is started from its favorable position (near N5 atom of FAD) which is true only if the $\mathrm{O}_{2}^{\bullet-}$ takes proton from the $\mathrm{N} 5 \mathrm{H}$. This indicates that the hydrogen bonding between the $\mathrm{N} 5 \mathrm{H}$ atom of radical or anionic FADH and $\mathrm{OO} 2$ atom of hydroxyl radical is the main electrostatic interaction between hydroxyl radical and FAD. If it forms $\mathrm{HO}_{2}^{\bullet}$ by taking proton from somewhere else of the protein or from solvent and is placed initially anywhere else than the favored position as shown in Figure 6, the $\mathrm{HO}_{2}^{\bullet}$ flies away from the C4a cavity. On the contrary, for the superoxide or triplet oxygen, the qualitative results that whether the oxygen species stays or leave the C4a cavity remain invariant on its initial position be it below the lumiflavin ring or in between the lumiflavin ring and adenine (in both case, close to the C4a atom). The binding location of the superoxide in the flavin pocket remain also the same. In order to investigate the possibility of formation of C4ahydroperoxyflavin within the limit of molecular dynamics simulations, the distance between N5 of lumiflavin ring and $0 \mathrm{O} 2$ of oxygen species is plotted in Figure 6, indicating the formation of a strong hydrogen bonding $\left[\mathrm{N} 5-\mathrm{H} . . \mathrm{O}_{2}\right]$ (with average donoracceptor distance $<3.0 \AA$ ) facilitating the formation of $\mathrm{FAD}^{\bullet-}+$ $\mathrm{HO}_{2}^{\bullet}$ as described in Figure 2. The low fluctuation of the ROS due to this hydrogen bonding also indicates towards possibility of formation of C4a-hydroperoxyflavin as suggested by other kinetic experimental studies. ${ }^{18,20,26}$. In the previous studies, the origin of the $\mathrm{H}^{+}$to form $\mathrm{HO}_{2}^{\bullet}$ from $\mathrm{O}_{2}^{\bullet-}$ was never discussed. From our MD simulation results, we propose that the N5 atom of FADH is most likely the proton donor to form the hydroperoxyl radical intermediate.

\subsection{Formation of superoxide and hydroxyl radicals}

Recent experiments have been able to detect superoxide and other ROS inside cryptochromes ${ }^{18-21}$. In this section, we discuss on the possibility of formation of ROS from electronic structure theory, in order to determine the oxidation state of oxygen.

The generation of superoxide implies an electron transfer from radical/anionic flavin to triplet oxygen. From a molecular orbital picture, the transferred electron is initially located on a singly occupied molecular orbital (SOMO) of radical FAD, which is of $\pi^{*}$
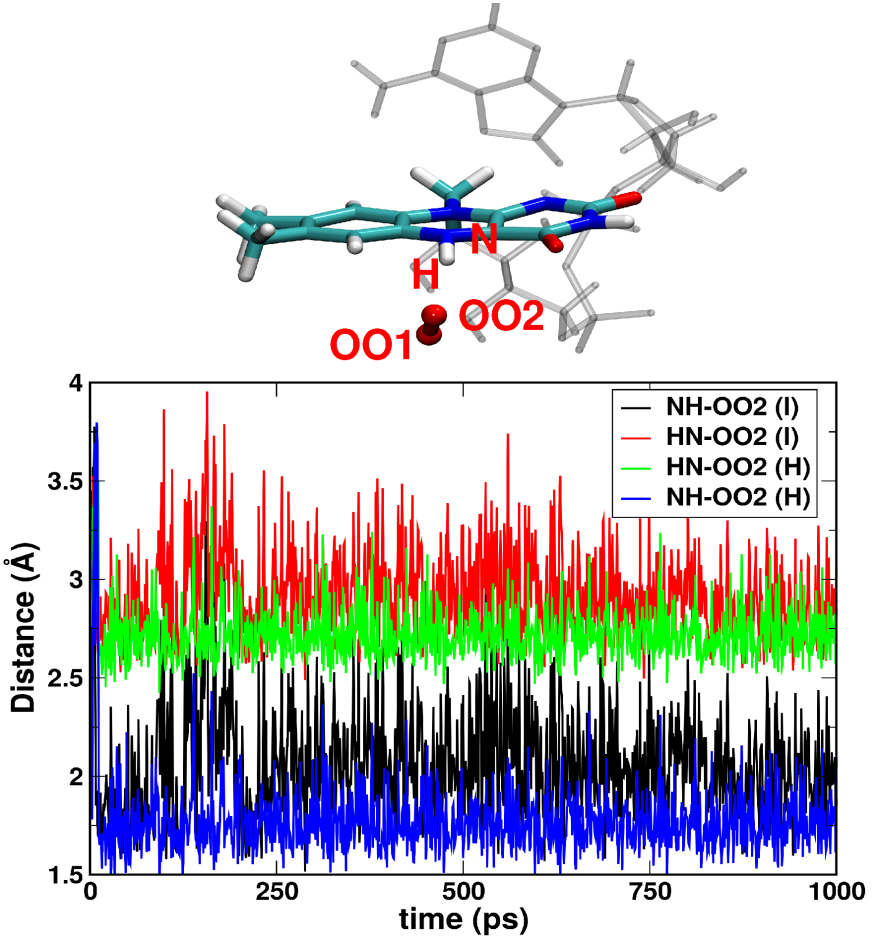

Fig. $6 \mathrm{HN} 5-\mathrm{OO} 2$ and $\mathrm{N} 5 \mathrm{H}-\mathrm{OO} 2$ distances for a typical trajectory for ${ }^{2}\left[\mathrm{FADH}^{+}-\mathrm{O}_{2}^{\bullet-}\right](\mathrm{H})^{3}\left[\mathrm{FADH}^{\bullet}-\mathrm{O}_{2}^{\bullet-}\right]$ system (I) indicating strong tendency to form $\mathrm{HO}_{2}^{*}$ radical. On top one typical structure (indicating the position of $\mathrm{O}_{2}^{*-}$ with respect to the $\mathrm{N} 5$ atom of $\mathrm{FADH}$ ) out of thousands is shown.

character residing between the C4a-N5 atoms. This electron will be transferred to the highest occupied molecular orbital (HOMO) of ${ }^{3} \mathrm{O}_{2}$, which consists of $\pi_{x}^{*}$ and $\pi_{y}^{*}$ orbitals each containing one electron. These orbitals are degenerate when oxygen is in an isotropic environment but, inside the binding pocket of cryptochrome, the electron will be transferred to the $\pi^{*}$ oxygen orbital that overlaps with the $\pi^{*}$ orbital of FAD.

Upon photo-absorption, an electron is added to the triplet $\left[\mathrm{FAD}_{o x}-\mathrm{O}_{2}\right]$ complex leading either to a doublet or quartet total spin, depending on the relative orientation of the independent spins of oxygen and radical FAD, see Figure 7 where the contributing configuration state functions for a radical $\mathrm{FAD}-\mathrm{O}_{2}$ system with minimal active space CAS $(3,3)$ are shown. In order to investigate the formation of superoxide, the energies of high and low spin states of different species involving radical oxygen are calculated using SA5-CASSCF+MS-CASPT2 method with larger active space of $(11,12)$ for the radical semi-quinones and $(12,12)$ for the reduced anion. These results are summarized in Table 1 . It should be noted that, after validating that the main interaction with $\mathrm{O}_{2}$ is localized in the lumiflavin ring, we considered only lumiflavin ring (instead of the full FAD cofactor) and oxygen in these calculations. The calculations are done on the structure of lumiflavinoxygen species extracted from the highest populated dmCry- $\mathrm{O}_{2}$ structure obtained using clustering analysis based on root mean square deviation implemented in Gromacs.

In Table 1, we report the three lowest energy states for 


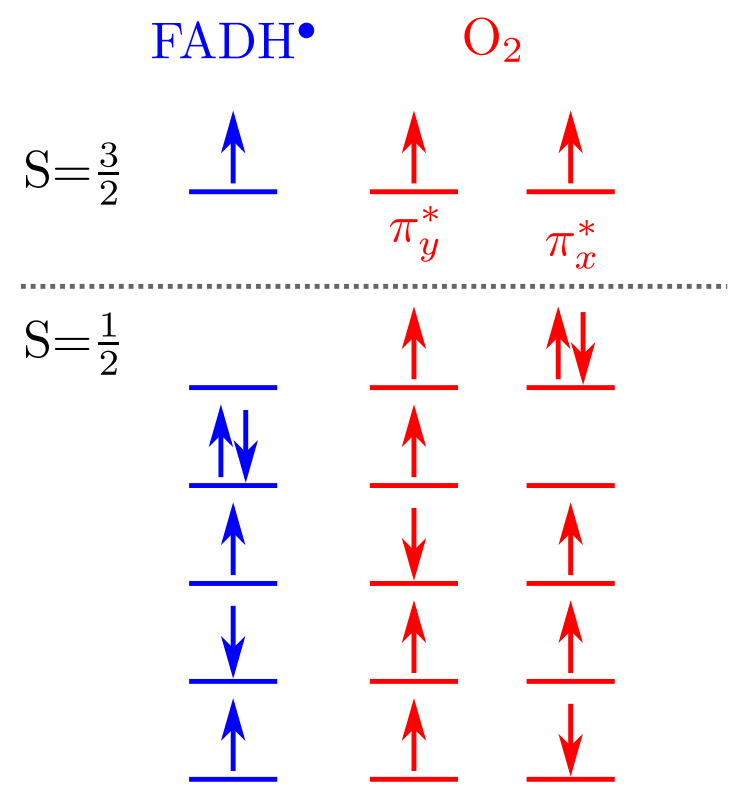

Fig. 7 Representation of the multiconfiguration wavefunction for a quartet $(S=3 / 2)$ and doublet $(S=1 / 2)$. The contributing configuration state functions for the ${ }^{4}\left[\mathrm{FADH}-\mathrm{O}_{2}\right]^{\bullet}(\mathrm{S}=3 / 2)$ and ${ }^{2}\left[\mathrm{FADH}-\mathrm{O}_{2}\right]^{\bullet}(\mathrm{S}=1 / 2)$ for the active space of $\operatorname{CAS}(3,3)$.

Table 1 SA5-CASSCF+MS-CASPT2 ground and excited state electronic energies for $\left[\mathrm{FADH}-\mathrm{O}_{2}\right]^{\bullet},\left[\mathrm{FAD}-\mathrm{HO}_{2}\right]^{\bullet},\left[\mathrm{FADH}-\mathrm{O}_{2}\right]^{-}$and $\left[\mathrm{FAD}-\mathrm{HO}_{2}\right]^{-}$ species. For comparison, the ground state has been set to 0.00 as reference for each species independently. The states (St) are labeled by their spin ( $S$ for singlet, $D$ for doublet, $T$ for triplet and $Q$ for quartet). Energies of first three states are shown for each spin. Spin multiplicity on oxygen species $\left(\mathrm{Mult}_{\mathrm{O}_{2}}\right.$ ) is shown based on Mulliken spin density. All energies are in $\mathrm{eV}$.

\begin{tabular}{crcrc} 
& {$\left[\mathrm{FADH}-\mathrm{O}_{2}\right]^{\bullet}$} & \multicolumn{3}{c}{$\left[\mathrm{FAD}_{o x}-\mathrm{HO}_{2}\right]^{\bullet}$} \\
\hline St & $\mathrm{E}_{e x}$ & $\mathrm{Mult}_{O_{2}}$ & $\mathrm{E}_{e x}$ & $\mathrm{Mult}_{O_{2}}$ \\
\hline D0 & 0.09 & 2.32 & 0.00 & 2.00 \\
D1 & 0.96 & 1.00 & 0.63 & 1.99 \\
D2 & 0.98 & 1.00 & 2.30 & - \\
Q0 & 0.00 & 2.94 & 2.27 & 2.00 \\
Q1 & 1.70 & 2.96 & 2.74 & 1.99 \\
Q2 & 3.35 & 2.03 & 3.28 & 2.00 \\
& & & & \\
& {$\left[\mathrm{FADH}-\mathrm{O}_{2}\right]^{-}$} & & $\left.\mathrm{FAD}_{o x}-\mathrm{HO}_{2}\right]^{-}$ & \\
\hline St & $\mathrm{E}_{e x}$ & $\mathrm{Mult}_{O_{2}}$ & $\mathrm{E}_{e x}$ & $\mathrm{Mult}_{O_{2}}$ \\
\hline S0 & 0.88 & - & 0.00 & - \\
S1 & 0.97 & - & 0.53 & - \\
S2 & 1.53 & - & 2.19 & - \\
T0 & 0.00 & 2.91 & 0.04 & 2.00 \\
T1 & 1.41 & 2.05 & 0.57 & 1.99 \\
T2 & 1.65 & 1.98 & 2.23 & 2.00 \\
\hline
\end{tabular}

each spin multiplicities of $\left[\mathrm{FADH}-\mathrm{O}_{2}\right]^{\bullet}$ (doublet or quartet) and $\left[\mathrm{FADH}-\mathrm{O}_{2}\right]^{-}$(singlet or triplet) species. In addition, we report the spin multiplicity of the oxygen fragment, extracted from the Mulliken analysis of the spin densities. First, we discuss the $\left[\mathrm{FADH}-\mathrm{O}_{2}\right]^{\bullet}$ form. The lowest energy state corresponds to the quartet ${ }^{4}\left[\mathrm{FADH}^{\bullet}-\mathrm{O}_{2}\right]$, in which oxygen is in a triplet spin state. However, the lowest energy doublet state is energetically close (only $0.09 \mathrm{eV}$ above Q0), suggesting that the doublet form can also exist $\left({ }^{2}\left[\mathrm{FADH}^{+}-\mathrm{O}_{2}^{\bullet-}\right]\right)$. In that case, the oxygen spin state could not be precisely determined from the Mulliken analysis, but still its multiplicity is closer to a doublet oxygen form. After the proton transfer between FAD and superoxide, the $\left[\mathrm{FAD}_{o x}-\mathrm{HO}_{2}\right]^{\bullet}$ complex is formed. The electronic structure calculations indicate that the doublet is the ground state, in which $\mathrm{HO}_{2}^{\bullet}$ has a doublet spin multiplicity. The lowest quartet state for this complex lays $2.26 \mathrm{eV}$ above the doublet. Second, we discuss the $\left[\mathrm{FADH}-\mathrm{O}_{2}\right]^{-}$ complex. The results for this complex suggest that the ground state is a triplet $\left[\mathrm{FADH}-\mathrm{O}_{2}\right]^{-}$, in which oxygen is a triplet. The radical superoxide is only formed in the first excited triplet state (T1) which exists as ${ }^{3}\left[\mathrm{FADH}^{\bullet}-\mathrm{O}_{2}^{\bullet-}\right]$ and is $\approx 1.4 \mathrm{eV}$ above the triplet ground state. On the other hand, in the lowest singlet state (which is $0.88 \mathrm{eV}$ above the triplet ground state) doublet superoxide is also formed. Upon a proton transfer, the complex $\mathrm{FAD}^{\bullet-}-\mathrm{HO}_{2}^{\bullet}$ is formed. For this complex, both the lowest singlet and triplets are almost degenerate $(\Delta E=0.038 \mathrm{eV})$ and both of them contains doublet oxygen species ${ }^{2} \mathrm{HO}_{2}^{\bullet}$. The $\mathrm{HO}_{2}^{\bullet}$ is formed in doublet not only in the ground state, but also for all other excited states of singlet and triplet multiplicity.

\subsection{The role of Trp420 and Cys416 in Drosophila melanogaster cryptochrome}

Trp420 is the closest tryptophan to the FAD. It has been reported that the closest tryptophan or a tryptophan triad containing the closest tryptophan is the electron donor that reduces FAD upon photon absorption, ${ }^{8}$ thus forming $\operatorname{TrpH}^{\bullet+}$ and subsequently Trp ${ }^{\bullet}$. Therefore, Trp420 is likely to be in radical form while considering cryptochrome with reduced FAD. To investigate the effect of Trp ${ }^{\bullet}$ on the position of $\mathrm{O}_{2}^{\bullet-}$, we ran further simulations for the ${ }^{2}\left[\mathrm{FADH}^{+}-\mathrm{O}_{2}^{\bullet-}\right]$ (a typical system where superoxide binds to lumiflavin) with the Mulliken charges for $\operatorname{Trp}{ }^{\bullet}$ and $\operatorname{TrpH}^{\bullet+}$ calculated at the same level of theory as discussed in Section 2. Figure 8a shows the comparison of the position of cluster of superoxide with respect to the $\mathrm{FADH}^{\bullet}$ and tryptophan for neutral Trp420 (red) and $\mathrm{TrpH}^{\bullet+}$ (blue) and $\operatorname{Trp}^{\bullet}$ (green). We observe that although the superoxide cluster is closer to the tryptophan for $\operatorname{TrpH}^{\bullet+}$ (blue) than for the neutral Trp420 (red) due to strong attractive electrostatic interactions between the negative superoxide and positively charged $\mathrm{NH}^{+}$, it is closer to the $\mathrm{FADH}^{\bullet}$ or almost at the same position for $\operatorname{Trp}^{\bullet}$ (green) with respect to the system with $\operatorname{Trp} 420$ (red).

To illuminate on the probable proton donor in dmCry after the photoreduction of FAD, the crystal structure of plant cryptochrome and dmCry is superimposed and it is observed that Cys416 in dmCry is in the same position of Asp396 of plant cryptochrome (Figure 8b left panel). Since Asp396 is reported to be 
the proton donor in plant cryptochrome ${ }^{8}$, by analogy, we propose Cys416 may act as a proton donor in dmCry. To further confirm this, an MD simulation run of $1 \mathrm{~ns}$ is performed for dmCry and the distance between the -SH of Cys416 and N5 of FAD is recorded and found them to be in close contact over the simulation time. Figure 3 shows the distance between the $\mathrm{H}$ of SH (Cys416) and N5 of the ISO(black)/N of the closest tryptophan, Trp420 (red) which indicates SH bond rotates freely- towards N5 of lumiflavin (to donate proton) and then towards NH of Trp420 (to get proton to compensate) which suggests that under favorable condition it is feasible that Cys416 can donate proton to semireduced anionic $\mathrm{FAD}$ and can get proton from tryptophan to compensate.

Cysteine has a $\mathrm{pK}_{a}$ value of 8.3 (in solution) which is close to physiological $\mathrm{pH}$ and therefore at the limit of acting as a proton donor. There have been studies in the literature on the possible role of cysteine as a proton donor in dmCry. Some spectroscopic studies suggested that the formation of $\mathrm{FADH}^{\bullet}$ in dmCry is hindered in physiological conditions, indicating that cysteine has a high $\mathrm{pK}_{a}$ value in protein. ${ }^{25,40}$ These would indicate that other residues in the cavity are responsible for the protonation of anionic radical semiquinonic flavin. At variance, Hong et al. used theoretical simulations to show that Cys416 pKa is 9.7 in protein. They conclude that Cys416 could act as the proton donor, despite being less efficient than aspartic acid. ${ }^{41}$ Based on their $\mathrm{pK}_{a}$ calculations, the difference (2.1) between the pKa of Asp396 in plant cryptochrome (7.6) and of Cys416 in dmCry (9.7) is smaller than the difference (4.4) of their $\mathrm{pK}_{a}$ in solution. Therefore, even though it is evident that FADH• is formed, it remains an open question whether Cys416 act as a possible proton donor or not. Cysteine can possibly act as a slow proton donor only at a higher $\mathrm{pH}$ value. A rigorous study based on constant $\mathrm{pH}$ molecular dynamics simulation would be necessary to confirm that.

\section{4 $\mathrm{FAD}-\mathrm{O}_{2}$ as alternative radical pair for magnetoreception}

Among the several reactions between FAD and oxygen species, there are four most important reactions involving a radical oxygen that is stabilized within the C4a cavity. Some of these reactions are also suggested to contribute to radical pair mechanism of magnetoreception by experimental spectral data ${ }^{18}$ ). The first two reactions correspond to the electron transfer from $\mathrm{FADH}^{\bullet}$ to $\mathrm{O}_{2}$ followed by proton transfer from $\mathrm{FADH}^{+}$to $\mathrm{O}_{2}^{\bullet-}$, that is,

$$
F A D H^{\bullet}+O_{2} \leftrightarrow F A D H^{+}+O_{2}^{\bullet-} \leftrightarrow F A D_{o x}+H O_{2}^{\bullet} .
$$

The third and four reactions correspond to the electron transfer from $\mathrm{FADH}^{-}$to $\mathrm{O}_{2}$ followed by proton transfer from $\mathrm{FADH}^{\bullet}$ to $\mathrm{O}_{2}^{\bullet}$, that is,

$$
F A D H^{-}+O_{2} \leftrightarrow F A D H^{\bullet}+O_{2}^{\bullet-} \leftrightarrow F A D^{\bullet-}+H O_{2}^{\bullet}
$$

There exist thus two radical pairs which may act as "alternative" radical pair and they are $\mathrm{FADH}^{\bullet}+\mathrm{O}_{2}^{\bullet-}$ and $\mathrm{FAD}^{\bullet-}+\mathrm{HO}_{2}^{\bullet}$. Still, there are few conditions to be satisfied for the radical pair mechanism for magnetoreception to work best: (i) the radical pair should be closely spaced for spin selective conversion, (ii) the electron nuclear hyperfine interaction should be weak, (iii)

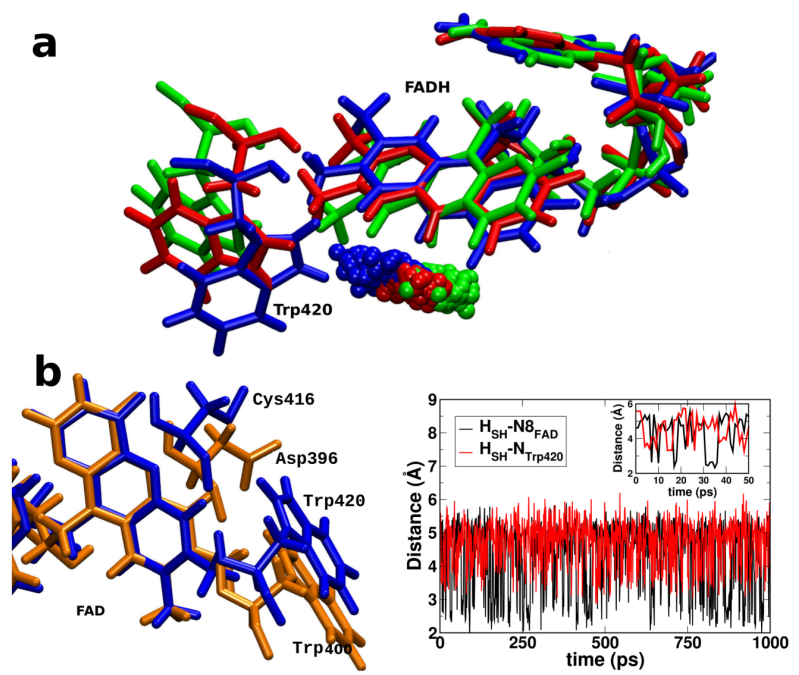

Fig. 8 (a) Comparison of the position of superoxide clusters for different redox and protonation states of tryptophan e.g. neutral Trp420 (red), $\operatorname{Trp}^{\bullet} \mathrm{H}^{+}$(blue) and $\operatorname{Trp} \bullet$ (green) . The structures are aligned with respect to the FADH ${ }^{\bullet}$. (b) Left: comparison of the core active site (containing FAD, Trp400/Trp420 and Asp396/Cys416 of plant (orange) and Drosophila melanogaster (blue) cryptochrome. Right : the distance between Hydrogen of $\mathrm{SH}$ (Cys416) and $\mathrm{N} 8$ of lumiflavin ring of FAD (black)/N of Trp420 (red). The inset shows the first 50 ps (zoomed) to detect the frequency of the $\mathrm{SH}$ bond rotation.

the spin relaxation of the radical should be slow, and (iv) the exchange and dipole-dipole spin couplings should be close to zero or mutually cancelling. ${ }^{42}$ With the results we have reported, we can qualitatively say that these conditions are satisfied for both radical pairs involving oxygen. Indeed, from our MD simulations we showed that $\mathrm{FADH}^{\bullet}-\mathrm{O}_{2}^{\bullet-}$ and $\mathrm{FAD}^{\bullet-}-\mathrm{HO}_{2}^{\bullet}$ stays within $2-3 \AA$. The hyperfine coupling condition is more easily satisfied by oxygen species radical pair than by tryptophan radical pair, since its ${ }^{16} \mathrm{O}$ and ${ }^{18} \mathrm{O}$ isotopes are not magnetic. Therefore, the hyperfine coupling of the radical pair is only due to $\mathrm{FADH}^{\bullet}$. For the spin relaxation, it is known that $\mathrm{O}_{2}^{\bullet-}$ has a fast spin relaxation kinetics ${ }^{18,21}$, but the presence of $\operatorname{Trp}^{\bullet}$ close to the radical pair (as shown in Figure 8) can act as scavenger to slow down the spin relaxation of oxygen species. In addition, the restricted mobility of radical pair (indicated by low fluctuation of oxygen species in Figure 3) due to strong electrostatic interactions can slow down the spin relaxation. Finally, from electronic structure calculations, we can estimate the exchange coupling strength as the difference between the low and the high spin lowest energies. From this criteria, we can conclude that only $\mathrm{FAD}^{\bullet-}-\mathrm{HO}_{2}^{\bullet}$ has a small exchange coupling $(\mathrm{J}=400 \mathrm{meV}$ ). From these results, we can conclude that $\mathrm{FAD}^{\bullet-}-\mathrm{HO}_{2}^{\bullet}$ is a good candidate for explaining magnetorecpetion via the "alternative" radical pair with oxygen.

\section{Conclusion}

In this work, we have systematically explored the fate of different oxygen species in the binding pocket of Drosophila melanogaster cryptochrome using atomistic molecular dynamics simulations, taking into account the charge and spin multiplicity of different FAD and oxygen forms that are generated upon blue light absorp- 
tion. Additionally, we performed electronic structure simulations of different $\mathrm{FAD}-\mathrm{O}_{2}$ radical pairs to clarify the oxidation state of oxygen in each of the complexes.

The molecular dynamics simulations suggest that radical oxygen species are strongly stabilized close to FAD due to strong attractive electrostatic interactions and hydrogen bonding. On the contrary, triplet oxygen species move out of the cavity when FAD is in radical or charged form. The electrostatic attractive forces between the radical oxygen species and FADH is dominated by the interaction between $\mathrm{N} 5 \mathrm{H}$ (of $\mathrm{FADH}^{\bullet}$ ) and $\mathrm{O}_{2}^{\bullet}$. The results also indicate to a strong tendency of formation of hydroperoxyl radical. The stability of hydroperoxyl radical near $\mathrm{C} 4 \mathrm{a}$ atom are in agreement with previous studies suggesting formation of C4ahydroperoxyflavin intermediate. ${ }^{26}$

We show that the formation of hydroperoxyl radical is possible by the proton transfer from the proton at N5 position in $\mathrm{FADH}^{-}$. Furthermore, we have found that the varying electrostatic field of radical or neutral tryptophan has negligible impact on the position of oxygen. Still, the oxygen species are closer to the cationic $\mathrm{TrpH}^{\bullet+}$ due to stronger electrostatic interactions. The four important systems that can lead to the formation of radical oxygen species are found to be ${ }^{2}\left[\mathrm{FADH}^{+}-\mathrm{O}_{2}^{\bullet-}\right],{ }^{2}\left[\mathrm{FAD}_{o x}-\mathrm{HO}_{2}^{\bullet}\right],{ }^{3}\left[\mathrm{FADH}^{\bullet}-\right.$ $\mathrm{O}_{2}^{\bullet-}$ ], ${ }^{3}\left[\mathrm{FAD}^{\bullet-}-\mathrm{HO}_{2}^{\bullet}\right]$.

The electronic structure analysis suggests that the radical $\mathrm{FADH}-\mathrm{O}_{2}$ system in the ground state is a quartet and exist as ${ }^{4}\left[\mathrm{FADH}^{\bullet}-\mathrm{O}_{2}\right]$. For this particular case, the ${ }^{2}\left[\mathrm{FADH}^{+}-\mathrm{O}_{2}^{\bullet-}\right]$ is only $\approx 0.1 \mathrm{eV}$ above the ground state and therefore it is possible that both spin states are formed in the protein. For the $\left[\mathrm{FADH}-\mathrm{O}_{2}\right]^{-}$ complex, the triplet radical pair ${ }^{3}\left[\mathrm{FADH}^{\bullet}-\mathrm{O}_{2}^{\bullet-}\right]$ is $1.5 \mathrm{eV}$ above the ground state ${ }^{3}\left[\mathrm{FADH}^{-}-\mathrm{O}_{2}\right]$ in which oxygen is in a triplet. The only radical pair in the ground state is ${ }^{3}\left[\mathrm{FAD}^{\bullet-}-\mathrm{HO}_{2}^{\bullet}\right]$ which has a small singlet-triplet energy gap.

In view of "alternative" radical pair, our MD results suggest that two probable radical pairs i.e. FADH ${ }^{\bullet}-\mathrm{O}_{2}^{\bullet-}$ and $\mathrm{FAD}^{\bullet-}-\mathrm{HO}_{2}^{\bullet}$. Among them, $\mathrm{FAD}^{\bullet-}-\mathrm{HO}_{2}^{\bullet}$ passes all criteria as discussed in subsection 3.4. Although, more investigation is necessary on the lifetime of the short-lived hydroperoxyl radical. The FADH ${ }^{\bullet}-\mathrm{O}_{2}^{\bullet-}$ radical pair passes all criteria except the small exchange coupling criteria. Further investigation is needed on whether the cancellation of dipole and exchange coupling is possible ${ }^{43}$. Environmental effects could also affect the order of states, but these are expected to be rather small in cryptochromes. ${ }^{44}$

To summarize, our results from this study focuses not only on the qualitative position of different oxygen species in the flavoprotein active site, but also detect its binding location, diffusion pathway and surrounding important residues depending on varying electrostatic interactions. These results are important not only in view of investigating the "alternative" radical pair mechanism of magnetoreception but also shed light on several other functionality of flavoprotein in general e.g. ROS production, antioxidant defence. The results from our study (e.g. the specific binding location, diffusion pathway of oxygen species and surrounding residues with strong interactions) can help designing mutant flavoprotein to accelerate ROS production as well as to accelerate anti-oxidant defence mechanism.

\section{Acknowledgments}

The authors acknowledge financial support by the "Agence Nationale pour la Recherche" through the project BIOMAGNET (ANR-16CE29-0008-01). This work was granted access to the HPC resources of Aix-Marseille Université financed by the project Equip@Meso (ANR-10-EQPX-29-01) of the program «Investissements d'Avenir» supervised by the Agence Nationale de la Recherche.

\section{References}

1 V. Massey, FASEB J., 1995, 9, 473-475.

2 I. Chaves, R. Pokorny, M. Byrdin, N. Hoang, T. Ritz, K. Brettel, L. O. Essen, G. T. J. van der Horst, A. Batschauer and M. Ahmad, Annu. Rev. Plant Bio., 2011, 62, 335-364.

3 A. Sancar, Chem. Rev., 2003, 103, 2203-2237.

4 P. Sampathkumar, S. Turley, J. E. Ulmer, R. H. G., C. H. Sibley and W. G. Hol, J. Mol. Biol., 2005, 352, 1091-1104.

5 H. Myllykallio, G. Lipowski, D. Leduc, J. Filee, P. Forterre and U. Liebl, Science, 2002, 297, 105-107.

6 E. Gross, K. D. B., C. A. Kaiser and D. Fass, Cell, 2004, 117, 601-610.

7 K. R. Marshall, M. Gong, L. Wodke, J. H. Lamb, D. J. Jones, P. B. Farmer, N. S. Scrutton and A. W. Munro, J. Biol. Chem., 2005, 280, 30735-30740.

8 I. A. Solovy'ov, T. Domratcheva, A. R. Moughal Shahi and K. Schulten, J. Am. Chem. Soc., 2012, 134, 18046-18052.

9 C. T. Rodgers and P. Hore, Proc. Nat. Acad. Sci., 2009, 106, 353-360.

10 T. Ritz, S. Adem and K. Schulten, BioPhys. J., 2000, 78, 707718.

11 Y.-T. Kao, C. Saxena, T.-F. He, L. Guo, L. Wang, A. sancar and D. Zhong, J. Am. Chem. Soc., 2008, 130, 13132-13139.

12 F. Cailliez, P. Müller, M. Gallois and A. de la Lande, J. Am. Chem. Soc., 2014, 136, 12974-12986.

13 R. J. Gegear, L. E. Foley, A. Casselman and S. M. Reppert, Nature, 2009, 463, 804-807.

14 T. Ritz, R. Wiltschko, P. Hore, C. T. Rodgers, K. Stapput, P. Thalau, C. R. Timmel and W. Wiltschko, Biophys. J., 2009, 96, 3451-3457.

15 N. Christine, D. Susanne, S. Katrin, A. Margaret, P. Leo, W. Wolfgang and W. Roswitha, J. Royal Soc. Interface, 2013, 10, 20130638.

16 D. R. Kattnig, J. Phys. Chem. B, 2017, 121, 10215-10227.

17 D. R. Kattnig and P. J. Hore, Sci. Rep., 2017, 7, 11640-11650.

18 P. Müller and M. Ahmed, J. Biol. Chem., 2011, 286, 2103321040.

19 L.-D. Arthaut, N. Jourdan, A. Mteyrek, M. Procopio, M. ElEsawi, A. d'Harlingue, P.-E. Bouchet, J. Witczak, T. Ritz, A. Klarsfeld, S. Birman, R. J. Usselman, U. Hoecker, C. F. Martino and M. Ahmad, PLOS ONE, 2017, 12, 1-15.

20 L. van Wilderen, G. Silkstone, M. Mason, J. J. van Thor and M. T. Wilson, FEBS open bio, 2015, 5, 885-892.

21 H. J. Hogben, O. Efimova, N. Wagner-Rundell, C. R. Timmel and P. J. Hore, Chem. Phys. Lett., 2009, 480, 118-122. 
22 R. Baron, C. Riley, P. Chenprakhon, K. Thotsaporn, R. T. Winter, A. Alfieri, F. Forneris, W. J. H. van Berkel, P. Chaiyen, M. W. Fraaije, A. Mattevi and J. A. McCammon, Proc. Nat. Acad. Sci., 2009, 106, 10603-10608.

23 B. Giovani, M. Byrdin, M. Ahmad and K. Brettel, Nat. Struct. Biol., 2003, 10, 489-490.

24 R. Banerjee, E. Schleicher, S. Kacprzak, J. P. Bouly, M. Picot, W. Wu, A. Berndt, E. Wolf, R. Bittl and M. Ahmad, J. Biol. Chem., 2007, 282, 14916-14922.

25 A. Berndt, T. Kottke, H. Breitkreuz, R. Dvorsky, S. Hennig, . Alexander, M and E. Wolf, J. Biol. Chem., 2007, 282, 1301113021.

26 V. Massey, J. Biol. Chem., 1994, 269, 22459-22462.

27 J.-D. Chai and M. Head-Gordon, Phys. Chem. Chem. Phys., 2008, 10, 6615-6620.

28 M. M. Francl, W. J. Pietro, W. J. Hehre, J. S. Binkley, M. S. Gordon, D. J. DeFrees and J. A. Pople, J. Chem. Phys, 1982, 77, 3654-3665.

29 H. J. C. Berendsen, D. van der Spoel and R. van Drunen, Comput. Phys. Commun., 1995, 91, 43-56.

30 A. D. J. Mackerell, M. Feig and C. L. r. Brooks, J. Comput. Chem., 2004, 25, 1400-1415.

31 P. Bjelkmar, P. Larsson, M. A. Cuendet, B. Hess and E. Lindahl, J. Chem. Theo. Comput., 2010, 6, 459-466.

32 K. Vanommeslaeghe, E. Hatcher, C. Acharya, S. Kundu, S. Zhong, J. Shim, E. Darian, O. Guvench, P. Lopes, I. Vorobyov and A. D. MacKerell (Jr.), J. Comp. Chem., 2010, 31, 671-690.

33 M. J. Frisch, G. W. Trucks, H. B. Schlegel, G. E. Scuseria, M. A. Robb, J. R. Cheeseman, G. Scalmani, V. Barone, G. A. Petersson, H. Nakatsuji, X. Li, M. Caricato, A. V. Marenich, J. Bloino, B. G. Janesko, R. Gomperts, B. Mennucci, H. P. Hratchian, J. V. Ortiz, A. F. Izmaylov, J. L. Sonnenberg, D. WilliamsYoung, F. Ding, F. Lipparini, F. Egidi, J. Goings, B. Peng, A. Petrone, T. Henderson, D. Ranasinghe, V. G. Zakrzewski, J. Gao, N. Rega, G. Zheng, W. Liang, M. Hada, M. Ehara, K. Toyota, R. Fukuda, J. Hasegawa, M. Ishida, T. Nakajima, Y. Honda, O. Kitao, H. Nakai, T. Vreven, K. Throssell, J. A. Montgomery, Jr., J. E. Peralta, F. Ogliaro, M. J. Bearpark, J. J. Heyd, E. N. Brothers, K. N. Kudin, V. N. Staroverov, T. A. Keith, R. Kobayashi, J. Normand, K. Raghavachari, A. P. Ren- dell, J. C. Burant, S. S. Iyengar, J. Tomasi, M. Cossi, J. M. Millam, M. Klene, C. Adamo, R. Cammi, J. W. Ochterski, R. L. Martin, K. Morokuma, O. Farkas, J. B. Foresman and D. J. Fox, Gaussian 16 Revision A.01, 2016, Gaussian Inc. Wallingford CT.

34 A. D. MacKerell (Jr.), D. Bashford, M. Bellott, R. L. R. L. Dunbrack (Jr), J. D. Evanseck, M. J. Field, M. Fischer, J. Gao, H. Guo, S. Ha, D. Joseph-McCarthy, L. Kuchnir, K. Kuczera, F. T. K. Lau, C. Mattos, S. Michnick, T. Ngo, D. T. Nguyen, B. Prodhom, W. E. Reiher, B. Roux, M. Schlenkrich, J. C. Smith, R. R. Stote, J. Straub, M. Watanabe, J. WiÃşrkiewiczKuczera, D. Yin and M. Karplus, J. Phys. Chem. B, 1998, 102, 3586-3616.

35 F. Autenreith, J. Tajkhorshid, E.and Baudry and Z. LutheySchulten, J. Comp. Chem., 2004, 25, 1613-1622.

36 B. O. Roos, V. Veryazov and P.-O. Widmark, Theor. Chem. Acc., 2004, 111, 345-351.

37 F. Aquilante, J. Autschbach, R. K. Carlson, L. F. Chibotaru, M. G. Delcey, L. De Vico, I. Fdez. GalvÃąn, N. Ferrễl, L. M. Frutos, L. Gagliardi, M. Garavelli, A. Giussani, C. E. Hoyer, G. Li Manni, H. Lischka, D. Ma, P. Ã. Malmqvist, T. MÃijller, A. Nenov, M. Olivucci, T. B. Pedersen, D. Peng, F. Plasser, B. B. Pritchard, M. Reiher, I. Rivalta, I. Schapiro, J. SegarraMartí, M. Stenrup, D. G. Truhlar, L. Ungur, A. Valentini, S. Vancoillie, V. Veryazov, V. P. Vysotskiy, O. Weingart, F. Zapata and R. Lindh, J. Comput. Chem., 2016, 37, 506.

38 N. Baker, D. Sept, S. Joseph, M. J. Holst and M. J. A., Proc. Nat. Acad. Sci., 2001, 98, 10037-10041.

39 W. Humphrey, A. Dalke and K. Schulten, J. Molec. Graphics, 1996, 14, 33-38.

40 B. Paulus, C. Bajzath, F. Melin, L. Heidinger, V. Kromm, C. Herkersdorf, U. Benz, L. Mann, P. Stehle, P. Hellwig, S. Weber and E. Schleicher, FEBS Journal, 2015, 282, 3175-3189.

41 G. Hong and R. Pachter, J. Phys. Chem. B, 2015, 119, 38833892.

42 P. J. Hore, Annu. Rev. BioPhys., 2016, 45, 299-344.

43 O. Efimova and P. J. Hore, BioPhys. J., 2008, 94, 1565-1574.

44 E. Cannuccia, O. Pulci, R. D. Sole and M. Cascella, Chem. Phys., 2011, 389, 35 - 38. 